OPEN ACCESS

Edited by:

Tianming Liu,

University of Georgia, United States

Reviewed by:

Feng Liu,

Tianjin Medical University General

Hospital, China

Kaiming $L i$

Sichuan University, China

${ }^{*}$ Correspondence:

Xia Wu

wuxia@bnu.edu.cn

Li Yao

yaoli@bnu.edu.cn

Received: 15 September 2017 Accepted: 19 December 2017

Published: 09 January 2018

Citation:

Li Q, Wu X, Xu L, Chen K, Yao L and Alzheimer's Disease Neuroimaging Initiative (2018) Classification of Alzheimer's Disease, Mild Cognitive Impairment, and Cognitively Unimpaired Individuals Using Multi-feature Kernel Discriminant Dictionary Learning

Front. Comput. Neurosci. 11:117. doi: 10.3389/fncom.2017.00117

\section{Classification of Alzheimer's Disease, Mild Cognitive Impairment, and Cognitively Unimpaired Individuals Using Multi-feature Kernel Discriminant Dictionary Learning}

\author{
Qing $\mathrm{Li}^{1}$, Xia $W u^{1,2 *}$, Lele $\mathrm{Xu}{ }^{1}$, Kewei Chen ${ }^{3}$, Li Yao ${ }^{1,2 *}$ and \\ Alzheimer's Disease Neuroimaging Initiative \\ ${ }^{1}$ Department of Electronics, College of Information Science and Technology, Beijing Normal University, Beijing, China, ${ }^{2}$ State \\ Key Laboratory of Cognitive Neuroscience and Learning, Beijing Normal University, Beijing, China, ${ }^{3}$ Banner Alzheimer's \\ Institute and Banner Good Samaritan PET Center, Phoenix, AZ, United States
}

Accurate classification of either patients with Alzheimer's disease (AD) or patients with mild cognitive impairment $(\mathrm{MCl})$, the prodromal stage of $A D$, from cognitively unimpaired (CU) individuals is important for clinical diagnosis and adequate intervention. The current study focused on distinguishing $\mathrm{AD}$ or $\mathrm{MCl}$ from $\mathrm{CU}$ based on the multi-feature kernel supervised within-Class-similar discriminative dictionary learning algorithm (MKSCDDL), which we introduced in a previous study, demonstrating that MKSCDDL had superior performance in face recognition. Structural magnetic resonance imaging (sMRI), fluorodeoxyglucose (FDG) positron emission tomography (PET), and florbetapir-PET data from the Alzheimer's Disease Neuroimaging Initiative (ADNI) database were all included for classification of AD vs. CU, MCl vs. CU, as well as AD vs. $\mathrm{MCl}$ (113 AD patients, $110 \mathrm{MCl}$ patients, and $117 \mathrm{CU}$ subjects). By adopting MKSCDDL, we achieved a classification accuracy of $98.18 \%$ for $A D$ vs. $\mathrm{CU}, 78.50 \%$ for $\mathrm{MCl}$ vs. $\mathrm{CU}$, and $74.47 \%$ for $A D$ vs. $\mathrm{MCl}$, which in each instance was superior to results obtained using several other state-of-the-art approaches (MKL, JRC, mSRC, and mSCDDL). In addition, testing time results outperformed other high quality methods. Therefore, the results suggested that the MKSCDDL procedure is a promising tool for assisting early diagnosis of diseases using neuroimaging data.

Keywords: Alzheimer's disease (AD), mild cognitive impairment (MCl), multimodal imaging, multiple kernel dictionary learning

\section{INTRODUCTION}

Alzheimer's disease (AD) is a complex multifactorial neurodegenerative disorder and is the most common type of dementia, defined by extensive neuronal and synapses loss (Tan et al., 2013; Gao et al., 2016). Recent study has shown that AD has high prevalence of an estimated 40 million patients worldwide (Selkoe and Hardy, 2016). Mild cognitive impairment (MCI) has been generally viewed as an intermediate state between normal aging and 




GRAPHICAL ABSTRACT |

the onset of AD (Petersen et al., 2001; Garcés et al., 2014). Thus, $\mathrm{AD}$ and $\mathrm{MCI}$, the transitional stage between the healthy aging and dementia, which commonly characterized by slight cognitive deficits but largely intact activities of daily living (Petersen, 2004; Wei et al., 2016), have been greatly interested.

It has been shown that the neuroimaging data, including structural magnetic resonance imaging (sMRI) (Wee et al., 2011; Zhou et al., 2011), functional MRI (fMRI) (Suk et al., 2013), fluorodeoxyglucose positron emission tomography (FDG-PET) (Sanabria-Diaz et al., 2013), and amyloid PETs, such as Pittsburgh compound B (PiB-PET) (Zhang et al., 2014), florbetapir-PET (Saint-Aubert et al., 2013), can be used to discriminate AD or MCI with promising results when each modality is used individually and separately. It has been speculated that different neuroimaging tool provides complementary information, which, when combined, can be more powerful for diagnosis of $\mathrm{AD}$ or MCI (Liu et al., 2014b; Suk et al., 2015; Wang et al., 2016) and combining these potentially complementary information from various modalities would produce more powerful classifiers (Zhang et al., 2012a; Xu et al., 2015).

Several classification methods of combining multi-modality data have been used to classify $\mathrm{AD}$ or MCI from CU. For example, a weighted multiple kernel learning (MKL) model has been proposed to classify AD or MCI based on combining different modalities (Wee et al., 2012; Zhang et al., 2012b; Liu et al., 2014b). A joint regression and classification (JRC) algorithm was also introduced and has been indicated to diagnosis $\mathrm{AD}$ or MCI effectively based on multi-modalities data (Zhu et al., 2014a,b). A weighted multimodality sparse representation-based classification (mSRC) was developed and applied for discriminating AD or MCI based on multi-modalities (Xu et al., 2015). Recently, a multimodal discriminative dictionary learning (mSCDDL) (Li et al., 2017) algorithm has been proposed for classifying $\mathrm{AD}$ or MCI efficiently, which was a weighted multi-modality way extended from supervised within-Class-similarity discriminative dictionary learning (SCDDL), a robust and efficient machine learning method for facial recognition by $\mathrm{Xu}$ et al (Xu et al., 2016).

SCDDL was a discriminant dictionary learning (DL), which combined the classification error term and the
within-Class-similarity in the objection function of DL scheme (Xu et al., 2016). Recently, SCDDL was extended to a kernel framework, due to MKL algorithm has been suggested to be effective for feature fusion (Gönen and Alpaydin, 2011), named as multi-feature kernel SCDDL (MKSCDDL) and has been indicated to be an efficient tool in face recognition ( $\mathrm{Wu}$ et al., 2017).

In this study, MKSCDDL was examined for its robustness and efficiency of classification accuracy for $\mathrm{AD}$ or $\mathrm{MCI}$ with CU, based on three modalities data i.e., sMRI, FDGPET and florbetapir-PET. Our experimental results indicated that the MKSCDDL method combined multi-modalities could outperform SCDDL with each modality data alone, and achieve better or comparable classification performance, compared with some other state-of-the-art multi-modality classification algorithms, including MKL (Zhang et al., 2011), JRC (Zhu et al., 2014a), mSRC (Xu et al., 2015), and mSCDDL (Li et al., 2017).

\section{IMAGE PREPROCESSING}

In this work, we used data from the Alzheimer's Disease Neuroimaging Initiative (ADNI) for performance evaluation. The ADNI was launched in 2003 by the National Institute on Aging (NIA), the National Institute of Biomedical Imaging and Bioengineering (NIBIB), the Food and Drug Administration (FDA), private pharmaceutical companies, and non-profit organizations, as a 5-year public-private partnership. For up-todate information, see http://www.adni-info.org.

\section{Subjects}

In this paper, 113 patients with $\mathrm{AD}, 110$ patients with MCI and $117 \mathrm{CU}$ with the age ranged from 55 to 99 years were included. All the data, including the sMRI, FDG-PET, and florbetapir-PET, were downloaded from ADNI 1, ADNI GO, or ADNI 2. For each subject, the data-acquisition interval of the three modalities was within four months. Moreover, the subjects were matched in terms of age, the years of education and gender. The subjects we selected satisfied the following criteria: (1) The MMSE score of each AD subject was between 20 and 26, with a CDR of 0.5 or 1.0. The AD group did not significantly differ with respect to the presence of APOE4 alleles from the MCI group $(p=0.765)$, 
but had significantly lower MMSE scores (compared with CU group, $p=1.24 \times 10^{-90}$; MCI group, $p=1.61 \times 10^{-40}$ ) and a different presence of APOE4 alleles compared with the CU group $(p=0.014)$. (2) The MMSE score of each MCI subject was between 24 and 30 , and the CDR was 0.5 . The MCI group had significantly lower MMSE scores $\left(p=4.69 \times 10^{-31}\right)$ and a different presence of APOE4 alleles $\left(p=7.34 \times 10^{-04}\right)$ compared with CU group. (3) The MMSE score of each CU was between 26 and 30 and their CDR was 0.0. Table 1 shows the demographic information of the subjects.

\section{Image Processing}

Images were preprocessed using the VBM8 (Voxel-Based Morphometry 8) Toolbox (http://dbm.neuro.uni-jena.de/ vbm8/) in SPM8 (Statistical Parametric Mapping 8) (http:// www.fil.ion.ucl.ac.uk/spm/) that running on MATLAB 2010b (The MathWorks, Inc., Sherborn, MA, USA). Based on adaptive maximum posterior and partial volume estimation, every structural image was segmented into rigid-body-aligned gray matter (GM), white matter (WM) and cerebrospinal fluid (CSF) for each subject (Rajapakse et al., 1997; Tohka et al., 2004). Spatially adaptive non-local approach was applied to improve the segmentation. The diffeomorphic anatomical registration through exponential lie algebra (DARTEL) protocol (Ashburner, 2007) in which template creation and image registration were performed to normalize the gray-matter images iteratively by using a diffeomorphic anatomical registration.

All FDG-PET and florbetapir-PET images were co-registered with each individual's sMRI using a rigid body transformation, and subsequently warped to the cohort-specific DARTEL template. Then, the standard uptake value ratio (SUVr) image was calculated for each FDG-PET image and florbetapirPET image; reference masks for quantification were defined relative to the whole brain (Langbaum et al., 2009; Sabbagh et al., 2015) or cerebellum (Reitan, 1958; Camus et al., 2012), respectively.

Then, based on the Automated Anatomical Labeling (AAL) (Tzourio-Mazoyer et al., 2002), 90 regions of interest (ROIs) (45 for each hemisphere; Table S1) were obtained. The feature of sMRI, FDG-PET, and florbetapir-PET were got by averaging the corresponding value of mean volume of GM, SUVr values of

TABLE 1 | Demographic information of the subjects, $p$-value was obtained using one-way ANOVA to the $\mathrm{AD}, \mathrm{MCl}$, and $\mathrm{CU}$ groups.

\begin{tabular}{lcccc}
\hline & AD $(\boldsymbol{n}=\mathbf{1 1 3})$ & $\mathbf{M C l}(\boldsymbol{n}=\mathbf{1 1 0})$ & $\mathbf{C U}(\boldsymbol{n}=\mathbf{1 1 7})$ & $\boldsymbol{p}$-value \\
\hline Gender & $62 \mathrm{M} / 51 \mathrm{~F}$ & $59 \mathrm{M} / 51 \mathrm{~F}$ & $62 \mathrm{M} / 55 \mathrm{~F}$ & 0.96 \\
Age & $75.6 \pm 7.6$ & $75.2 \pm 7.8$ & $75.4 \pm 7.0$ & 0.94 \\
EDU & $16.10 \pm 3.00$ & $16.57 \pm 2.76$ & $16.44 \pm 2.41$ & 0.65 \\
MMSE & $22.4 \pm 2.2$ & $27.4 \pm 1.9$ & $28.9 \pm 1.3$ & $7.75 \times 10^{-75}$ \\
CDR & $0.8 \pm 0.2$ & $0.5 \pm 0.0$ & $0.0 \pm 0.0$ & $7.00 \times 10^{-151}$ \\
APOE4 (\%) & 50.00 & 52.73 & 24.49 &
\end{tabular}

$A D$, Alzheimer's disease; $M C l$, mild cognitive impairment; $C U$, cognitively unimpaired; $M$, male; F, female; MMSE, Mini-Mental State Examination; CDR, Clinical Dementia Rating; EDU, years of education; APOE4, percentage of APOE4 alleles.
FDG-PET and florbetapir-PET from each ROI that all the voxels within the ROI of each subject.

\section{METHOD}

\section{Discriminant Dictionary Learning}

Suppose $n$ training samples with $d$-dimension from $k$ classes are represented by $A=\left[a_{1}, a_{2}, \ldots, a_{n}\right]=\left[A_{1}, \ldots, A_{l}, \ldots, A_{k}\right] \in$ $\mathfrak{R}^{d \times n}$, in which, column vector $a_{i}$ is the sample $i(i=1, \ldots, n)$, and submatrix $A_{j}$ consists of column vectors (samples) from class $j(j=1, \ldots, k)$, and there are $m$ atoms (each column of the dictionary can be viewed as an atom) in the corresponding dictionary $D=\left[d_{1}, d_{2}, \ldots, d_{m}\right] \in \mathfrak{R}^{d \times m}(m \leq n)$. The general supervised DL model can be denoted as follows:

$$
\begin{aligned}
& \langle D, \theta, X\rangle=\arg \min _{D, \theta, X}\|A-D X\|_{F}^{2}+\lambda_{1}\|X\|_{1}+\lambda_{\theta} g(\theta) \\
& \text { s.t. }\left\|d_{j}\right\|_{2}^{2}=1, \text { for all } j=1, \ldots, m
\end{aligned}
$$

where $\theta$ is the discriminative parameter and $g(\theta)$ represents the discriminative term, $X$ denotes the coding coefficients of training samples $A$ on the dictionary $D . g(\theta)$ here indicates the linear classification error function (like $\|H-W X\|_{F}^{2}$ in the DL methods of D-KSVD (Zhang and Li, 2010) and LC-KSVD (Jiang et al., 2013), where $H$ is the class label matrix and $W$ is a classifier).

For classification, the classifier learned with the dictionary may be optimal simultaneously, as in the DL algorithms that incorporate a linear classification error term (Zhang and Li, 2010). However, the inner-structure of representation coefficients between classes has not been considered in such approach. To further enhance the discriminant power of the dictionary, both the linear classifier and the direct restriction of within-Class scatter on coding coefficients in the above discriminant DL scheme in our previous study are indicated (Xu et al., 2016), which is referred to as the SCDDL algorithm.

\section{Supervised within-Class-Similar Discriminative Dictionary Learning}

Suppose $A=\left[A_{1}, \ldots, A_{l}, \ldots, A_{k}\right] \in \mathfrak{R}^{d \times n}$ denotes the $n d$-dimensional training samples from $k$ classes, $D \in$ $\mathfrak{R}^{d \times m}(m \leq n)$ is the discriminative dictionary with $m$ atoms that needs to be derived, and $X$ represents the coding coefficients of training samples $A$ on the dictionary $D$, denoted as $X=$ $\left[X_{1}, \ldots, X_{l}, \ldots, X_{k}\right] \in \Re^{m \times n}$, same as above. The SCDDL model can be written as follows:

$$
\begin{aligned}
\langle D, W, X\rangle= & \arg \min _{D, W, X}\|A-D X\|_{F}^{2}+\alpha\|H-W X\|_{F}^{2}+\beta\|W\|_{F}^{2} \\
& +\lambda_{1}\|X\|_{1}+\lambda_{2} \sum_{i=1}^{k}\left(\left\|X_{i}-M_{i}\right\|_{F}^{2}+\eta\left\|X_{i}\right\|_{F}^{2}\right) \\
\text { s.t. }\left\|d_{j}\right\|_{2}^{2}= & 1, \text { for all } j=1, \ldots, m
\end{aligned}
$$

where $\|\cdot\|_{F}^{2}$ represents the Frobenius norm. $\|A-D X\|_{F}^{2}$ is the reconstructed error term of the training samples $A$ on the newly constructed dictionary $D, \alpha\|H-W X\|_{F}^{2}+\beta\|W\|_{F}^{2}$ is the linear classification error term, and $\sum_{i=1}^{k}\left(\left\|X_{i}-M_{i}\right\|_{F}^{2}+\eta\left\|X_{i}\right\|_{F}^{2}\right)$ is 
the within-Class-similar term. $W \in \mathfrak{R}^{k \times m}$ is the parameter of the classifier; each column of $H \in \mathfrak{R}^{k \times m}$ is a vector, corresponds to one training sample with the form as $[0,0, \ldots, 1, \ldots, 0,0] \in \mathfrak{R}^{k}$, where 1 locates the corresponding class of the training sample; and each column of $M_{i}$ is the mean vector of the coefficients $X_{i}$ corresponding to class $i$. According to the elastic-net theory, the term $\left\|X_{i}\right\|_{F}^{2}$ combined with the term $\|X\|_{1}$ might make the solution of Equation (2) more stable (Zou and Hastie, 2005); and $\eta$ is set as $\eta=1$ for simplicity (Yang et al., 2014). Then Equation (2) can be written as:

$$
\begin{aligned}
\langle D, W, X\rangle= & \arg \min _{D, W, X}\|A-D X\|_{F}^{2}+\alpha\|H-W X\|_{F}^{2}+\beta\|W\|_{F}^{2} \\
& +\lambda_{1}\|X\|_{1}+\lambda_{2} \sum_{i=1}^{k}\left(\left\|X_{i}-M_{i}\right\|_{F}^{2}+\left\|X_{i}\right\|_{F}^{2}\right)
\end{aligned}
$$$$
\text { s.t. }\left\|d_{j}\right\|_{2}^{2}=1, \text { for all } j=1, \ldots, m
$$

The optimization process of Equation (3) has been discussed in our previous study (Xu et al., 2016). In SCDDL, the directly restricted within-Class-similar term makes the coding coefficients similar within one class and the linear classification error term selects the optimal classifier. This combination has been shown to improve the discriminative classification of the dictionary (Xu et al., 2016).

After obtaining the dictionary $D$ and classifier $W$ in the SCDDL model, the test samples can be finally classified.

For a given test sample $y$, the representation coefficient on $D$ is:

$$
x=\arg \min _{x}\left\|y-D_{x}\right\|_{2}^{2}+\lambda\|x\|_{1}
$$

where $\lambda$ is a scalar constant. The representation coefficient $x$ can be simply combined with the linear classifier $W$. Then the final identification of the test sample $y$ is obtained in the DL procedure with:

$$
\text { label }(y)=\arg \max _{l}\left\{W_{x}\right\}_{l}, l=1,2, \ldots, k
$$

where $\{\cdot\}_{l}$ represents the $l$-th element in the brace, $x$ contains discriminant information for classification.

\section{Multi-feature Kernel SCDDL (MKSCDDL)}

The SCDDL model is extended to a kernel framework for the further multi-feature fusion in our previous study ( $\mathrm{Wu}$ et al., 2017). Suppose $\phi(\cdot)$ is a mapping function from $R^{N}$ to a higher dimensional feature space. To avoid the explicit high-dimensional mapping procedure, mercer kernels could be helpful. The common mercer kernels include the linear kernel $k(x, y)=\langle x, y\rangle$, which equals to non-mapping; the Gaussian kernels $k(x, y)=\exp \left(-\frac{\|x-y\|^{2}}{c}\right)$; the polynomial kernels $k(x, y)=$ $(\langle x, y\rangle+c)^{d}$ ( $c$ and $d$ are parameters) and the sigmoid kernels $k(x, y)=\tanh \left(a\left(x^{T} y\right)+r\right)(a$ and $r$ are parameters) (Manevitz and Yousef, 2001; Hussain et al., 2011; Liu et al., 2013; Pham and Pagh, 2013; Dyrba et al., 2015).

The training samples $A$ and dictionary $D$ can be mapped to a higher dimensional space by a function of $\phi(\cdot)$, then $A$ and $D$ in the SCDDL model can be replaced by $\phi(A) \in R^{d_{\text {map }} \times n}$ and $\phi(D) \in R^{d_{\text {map }} \times m}$ ( $d_{\text {map }}$ is the dimensional number in the mapping space) respectively for the kernel SCDDL framework as follows:

$$
\begin{aligned}
\langle D, W, X\rangle= & \arg \min _{D, W, X}\|\phi(A)-\phi(D) X\|_{F}^{2}+\alpha\|H-W X\|_{F}^{2} \\
& +\beta\|W\|_{F}^{2}+\lambda_{1}\|X\|_{1} \\
& +\lambda_{2} \sum_{i=1}^{k}\left(\left\|X_{i}-M_{i}\right\|_{F}^{2}+\left\|X_{i}\right\|_{F}^{2}\right) \\
\text { s.t. }\left\|d_{j}\right\|_{2}^{2}= & 1, \text { for all } j=1, \ldots, m
\end{aligned}
$$

The dictionary can be represented by the training samples as Equation (7), according to the represented theorem (Schölkopf et al., 2001):

$$
\phi(D)=\phi(A) V
$$

where $V \in R^{n \times m}$ is the representation matrix. Equation (6) can be transformed to Equation (8) with Equation (7):

$$
\begin{aligned}
\langle V, W, X\rangle= & \arg \min _{V, W, X}\|\phi(A)-\phi(A) V X\|_{F}^{2}+\alpha\|H-W X\|_{F}^{2} \\
& +\beta\|W\|_{F}^{2}+\lambda_{1}\|X\|_{1}+\lambda_{2} \sum_{i=1}^{k}\left(\left\|X_{i}-M_{i}\right\|_{F}^{2}\right. \\
& \left.+\left\|X_{i}\right\|_{F}^{2}\right)
\end{aligned}
$$

The optimization process of Equation (8) has been discussed in our previous study (Wu et al., 2017). Then, the test sample $y$ and dictionary $D$ in Equation (4) can be replaced by $\phi(y) \in R^{d_{\text {map }}}$ and $\phi(A) V$ respectively as:

$$
x=\arg \min _{x}\|\phi(y)-\phi(A) V x\|_{2}^{2}+\lambda\|X\|_{1}
$$

where $\lambda$ is a scalar constant as above.

Let $T(x)=\min _{x}\|\phi(y)-\phi(A) V x\|_{2}^{2}$, then $T(x)$ can be simplified as:

$$
T(x)=\min _{x} \operatorname{tr}\left(x^{T} P x-2 x^{T} Q+S\right)
$$

where $P=V^{T} k(A, A) V, Q=V^{T} k(y, A)$, and $S=k(y, y)$.

Using the conclusions in previous study (Harandi and Salzmann, 2015), Equation (10) is equivalent to:

$$
T(x)=\arg \min _{x}\|\tilde{y}-\tilde{D} x\|_{2}^{2}
$$

where $\tilde{y}=\Sigma^{-\frac{1}{2}} U^{T} Q, \tilde{D}=\Sigma^{\frac{1}{2}} U^{T}$, and $U \Sigma U^{T}$ is the SVD of $P$ (Nguyen et al., 2012). Then Equation (9) can be denoted as:

$$
\langle x\rangle=\arg \min _{x}\|\tilde{y}-\tilde{D} x\|_{2}^{2}+\lambda_{1}\|X\|_{1}
$$

The convex problems in Equation (12) can be efficiently solved by plenty of tools such as the $L_{1}$-magic software package (Candes and Romberg, 2005), the GPSR package (Figueiredo et al., 2007) and the $L_{1}$-homotopy package (Asif and Romberg, 2010). 
Finally, the identification of the test sample $y$ can be employed using Equation (5) as follows:

$$
\text { label }(y)=\arg \max _{l}\left\{W_{x}\right\}_{l}, l=1,2, \ldots, k
$$

where the $\{\cdot\}_{l}$ represents the $l$-th element in the brace.

As it is shown in the MKL algorithm (Sonnenburg et al., 2006), suppose there are $J$ features for each sample, the kernel can be combined by convex combinations of $J$ kernels, i.e.,

$$
k(x, y)=\sum_{j=1}^{J} w_{j} k_{j}(x, y) w_{j} \geq 0, \quad \sum_{j=1}^{J} w_{j}=1
$$

where each sub-kernel $k_{j}$ corresponds to feature $j$.

So far, the kernels involved in the solution of Equation (12) can be replaced by Equation (13) for the multi-feature fusion of MKSCDDL. The combination coefficients can be simply set to be equal across all the features or optimized by crossvalidation on the training samples. The sub-kernels can be selected from linear kernel, polynomial kernels, Gaussian kernels and sigmoid kernels etc. After the substitution of the kernels involved in the solution of Equation (12), MKSCDDL is realized (Wu et al., 2017).

\section{Experimental Setting}

In MKSCDDL model and the classification scheme, there are several parameters need to be set, including the parameter $\alpha$ for the classification error term, $\lambda$ for the sparse coding term, $\lambda_{1}$ for the sparsity term, and $\lambda_{2}$ for the with-Class-similar term. Here, for simplify, $\alpha$ was set with $\alpha=1$ to make the contribution of the classification error equal (Xu et al., 2016). Furthermore, the parameter in the classification scheme $\lambda$ made a little effect in the experimental results. So, $\lambda$ was set with $\lambda=0.001$ in the experiment. For the parameters in the optimization model $\lambda_{1}$ and $\lambda_{2}$, the optimal values were searched from a small set of $\{0.001,0.005,0.01,0.05,0.1\}$ with a 5 -fold cross-validation on the training set (Wu et al., 2017). For the AD and CU data set: $\lambda_{1}=0.001, \lambda_{2}=0.1$. For the MCI and CU data set: $\lambda_{1}=$ $0.05, \lambda_{2}=0.05$. For the AD and MCI data set: $\lambda_{1}=0.05$, $\lambda_{2}=0.005$.
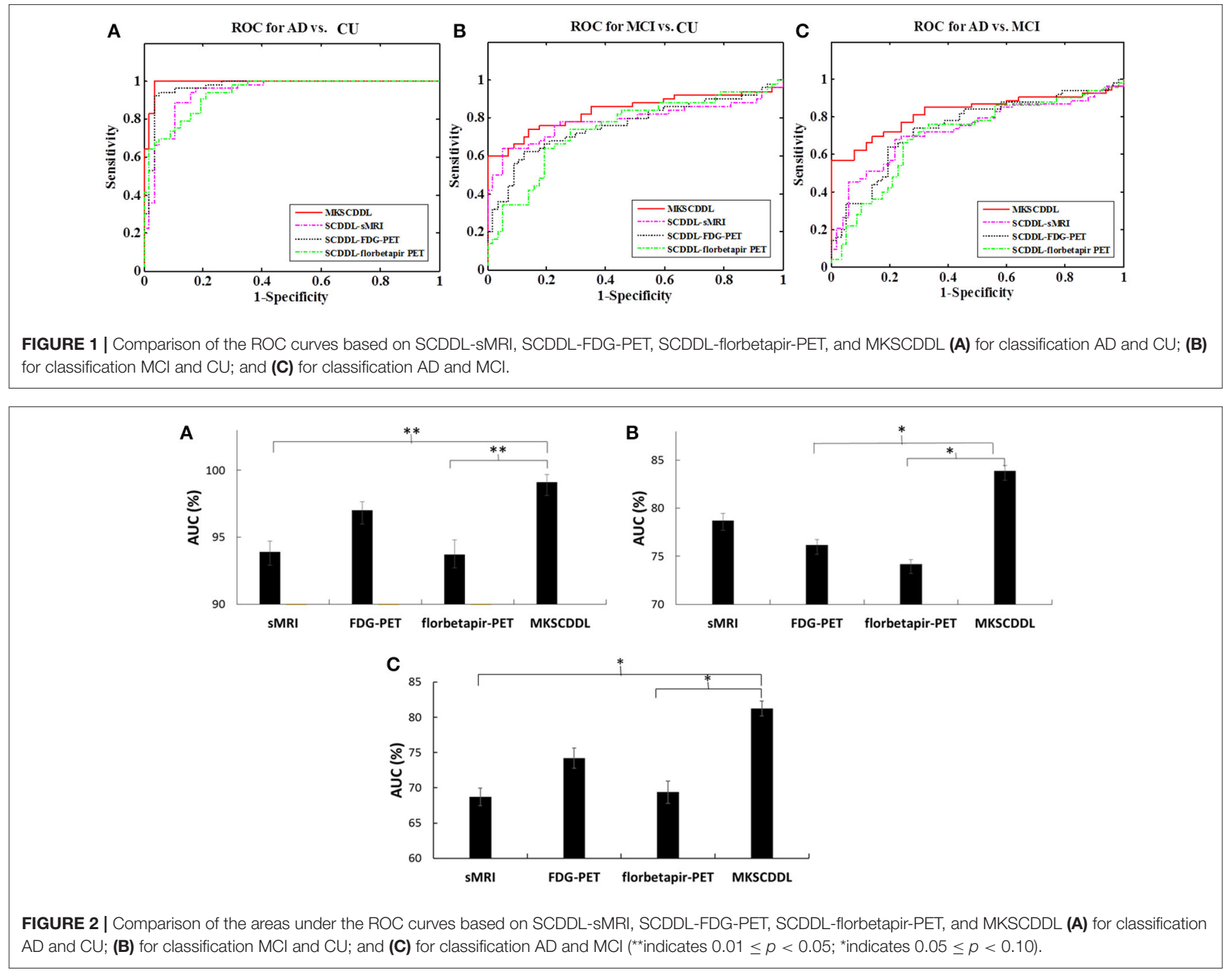
The dictionary size in MKSCDDL, mSCDDL, and SCDDL were set as 20 atoms (equivalent to 10 atoms for each class) for $\mathrm{AD} / \mathrm{CU}, \mathrm{MCI} / \mathrm{CU}$ and $\mathrm{AD} / \mathrm{MCI}$ classification; for MKL and JRC algorithms, all the training samples were trained for the model and classification; and for $\mathrm{mSRC}$, all the training samples were used as a dictionary.

In this study, linear kernel was employed for MKSCDDL in the experiment. The combining weight parameters of three modalities for MKSCDDL was derived based on grid search approach with the range of $[0,1]$ at a step size of 0.1 with a 5-fold cross-validation on training set (Zhang et al., 2011; Xu et al., 2015, 2016). Particularly, the combing weight parameters optimized corresponding to sMRI, FDG-PET and florbetapirPET for classifying $\mathrm{AD}$ from $\mathrm{CU}$ are $0.5,0.3$, and 0.2 ; for discriminating $\mathrm{MCI}$ from $\mathrm{CU}$ are $0.2,0.7$, and 0.1 ; for detecting $\mathrm{MCI}$ from $\mathrm{AD}$ are $0.3,0.6$, and 0.1 .

To evaluate the performance of all competing methods, their accuracy (the ratio of samples correctly classified among the test samples), sensitivity (the ratio of positive classes that were correctly identified), specificity (the ratio of negative classes that were accurately classified), and the areas under the Receiver Operating Characteristic (ROC) curves (AUC) were employed and compared in classification. For each group (AD, MCI, and $\mathrm{CU}$ ), samples (subjects) were divided randomly into training and test sets. Sixty samples were selected randomly as the training set, and the rest comprised the test set. The division process was then repeated five times for the results of means and standard deviations, which were reported in this paper. Then, a twosample t-test was carried out for each comparison pair to obtain the $p$-value.

In order to find the biomarkers for $\mathrm{AD}, \mathrm{MCI}$ and $\mathrm{CU}$ classification, the 90 features were ranked according to the significance of the two-sample $t$-test. Then, the classification accuracy with different number (from 1 to 90 ) of the ranked 90 features has been calculated based on MKSCDDL (Zhang et al., 2011; Xu et al., 2016).

\section{RESULTS AND DISCUSSIONS}

\section{Comparison with Single-Modality SCDDL}

The performance of using single-modality SCDDL (SCDDLSMRI, SCDDL-FDG-PET, and SCDDL-florbetapir-PET) and MKSCDDL (sMRI + FDG-PET + florbetapir-PET) were evaluated, as shown in Figures 1, 2 and Table 2, the MKSCDDL achieved higher accuracy in classifying $\mathrm{AD}, \mathrm{MCI}$, and $\mathrm{CU}$ than single-modality SCDDL methods.

For discriminating AD from CU, MKSCDDL achieved an accuracy of $98.18 \%$ (with $99.81 \%$ sensitivity and $96.49 \%$ specificity) that was much better than the best accuracy of $91.18 \%$ with single-modality method (using SCDDL-FDG-PET). Further, the comparison of the ROC curves for classification of $\mathrm{AD}$ and $\mathrm{CU}$ is shown in Figure 1A, and the comparison of AUCs is shown in Table 2. The ROC curve of MKSCDDL was closer to the top-left corner than that of SCDDL-FDG-PET, SCDDLflorbetapir-PET, and SCDDL-sMRI. The AUC of MKSCDDL was 0.991 , which was better than the single-modality methods $(\mathrm{AUC}=0.939, p=0.046$ for SCDDL-sMRI; $\mathrm{AUC}=0.937$, 
$p=0.028$ for SCDDL-florbetapir-PET; and AUC $=0.970$, $p=0.151$ for SCDDL-FDG-PET, which was not significant in validation, but was numerically greater) as shown in Figure 2A.

For classifying MCI from CU, MKSCDDL achieved an accuracy of $78.50 \%$ (with sensitivity of $76.00 \%$ and specificity of $81.06 \%$ ), which was greater than all three single-modality methods (the best classification accuracy was $72.50 \%$ when using SCDDL-FDG-PET). The comparison of the ROC curves for classification of $\mathrm{MCI}$ and $\mathrm{CU}$ are shown in Figure 1B and the comparison of AUCs is shown in Table 2. The ROC curve of MKSCDDL was closer to the top-left corner than that of SCDDL-sMRI, SCDDL-florbetapir-PET, and SCDDLFDG-PET. Further, based on the significance validation, MKSCDDL was significantly much better than the singlemodality methods with AUC, which was 0.839 for the multimodality method compared with that of the single-modality methods (AUC $=0.762, p=0.094$ for SCDDL-FDG-PET; $\mathrm{AUC}=0.742, p=0.076$ for SCDDL-florbetapir-PET; $\mathrm{AUC}=$ 0.787, $p=0.315$ for SCDDL-sMRI, which were numerically better, though were not significant in validation) as shown in Figure 2B.

For classifying AD from MCI, MKSCDDL achieved an accuracy of $74.47 \%$ (with sensitivity of $72.44 \%$ and specificity of $78.99 \%$ ), which was greater than all three single-modality methods (the best classification accuracy was $72.23 \%$ when using SCDDL-FDG-PET). The comparison of the ROC curves for classification of $\mathrm{AD}$ and MCI are shown in Figure $1 \mathrm{C}$ and the comparison of AUCs is shown in Table 2. The ROC curve of MKSCDDL was closer to the top-left corner than that of SCDDLsMRI, SCDDL-florbetapir-PET, and SCDDL-FDG-PET. Further, based on significant validation, MKSCDDL was significantly much better than the single-modality methods with AUC, which



FIGURE 3 | Comparison of the ROC curves based on JRC, MKL, mSRC, mSCDDL, and MKSCDDL (A) for classification AD and CU; (B) for classification MCl and
$\mathrm{CU}$; and (C) for classification AD and MCI.




was 0.791 for the multi-modality method compared with that of the single-modality methods (AUC $=0.687, p=0.091$ for SCDDL-sMRI; AUC $=0.694, p=0.107$ for SCDDL-florbetapirPET; and $\mathrm{AUC}=0.742, p=0.198$ for SCDDL-FDG-PET, which was numerically better, though were not significant in validation) as shown in Figure 2C.

The MKSCDDL achieved better classification accuracy and AUC for AD, MCI, and CU classification than the methods based on single-modality SCDDL (SCDDL-sMRI, SCDDL-FDG-PET, and SCDDL-florbetapir-PET), as seen in the results above, either statistically or numerically. The results we derived here were also consistent with those of other studies that have reported fusing multiple modalities could obtain better classification accuracy (Zhang et al., 2011; Westman et al., 2012; Xu et al., 2016).

Notably, on differentiating between MCI and CU, the classification specificity based on SCDDL-FDG-PET was $81.23 \%$, which was slightly higher than that based on MKSCDDL (81.06\%), whereas the classification sensitivity based on SCDDLFDG-PET (62.20\%) was much lower than that of MKSCDDL $(76.00 \%)$. Lower sensitivity with only marginally higher specificity (which could be due to random noise) would result in underdiagnosis. The MKSCDDL method had higher sensitivity and outstanding specificity that was comparable with that of SCDDL-FDG-PET, and much higher than that of the other methods. Therefore, the results suggest the feasibility of using MKSCDDL for neuroimaging classification tasks. These meant that the MKSCDDL method was much or slightly better than SCDDL-florbetapir-PET, SCDDL-sMRI and SCDDL-FDG-PET in differentiating $\mathrm{AD}$ or MCI from CU.

\section{Comparison with Several Other Multi-modality Methods}

The performance of using MKL, JRC, mSRC, mSCDDL, and MKSCDDL were evaluated and compared, including recognition rate, ROC curve and testing time. As shown in Figures 3-5 and
Table 3, the MKSCDDL achieved higher accuracy in classifying $\mathrm{AD}$ or MCI from CU than other multimodal methods, and outperforms in testing time.

For differentiating $\mathrm{AD}$ from $\mathrm{CU}$, MKSCDDL achieved an accuracy of $98.18 \%$ accuracy $\mathrm{CU}$ that was higher than MKL (93.64\%), JRC (94.55\%), mSRC (94.55\%), and mSCDDL (97.36\%). The comparison of the ROC curves for classification of $\mathrm{AD}$ and $\mathrm{CU}$ is shown in Figure $\mathbf{3 A}$ and the comparison of AUCs is shown in Table 3. The ROC curve of MKSCDDL was closer to the top-left corner than that of MKL, JRC, mSRC, and mSCDDL. The areas under the ROC curves for differentiation of $\mathrm{AD}$ and $\mathrm{CU}$ based on the five different methods are displayed in Figure 4A, in which the MKSCDDL method (AUC $=0.991$ ) performed equally well statistically or numerically better than the other three multi-modality methods (AUC $=0.963, p=0.095$ for $\mathrm{MKL} ; \mathrm{AUC}=0.971, p=0.291$ for JRC; $\mathrm{AUC}=0.978, p=0.429$ for $\mathrm{mSRC}$; and $\mathrm{AUC}=0.985, p=0.603$ for $\mathrm{mSCDDL}$ ). Figure 5 has shown the computational time for classification of per test sample with the corresponding methods. As shown, MKSCDDL consumed much less testing time than JRC $(p=0.007)$, mSRC $(p=0.010)$, and mSCDDL $(p=0.036)$, and was comparable with the MKL $(p=0.208)$ method.

For classifying MCI from CU, MKSCDDL achieved an accuracy of $78.50 \%$ (with sensitivity of $76.00 \%$ and specificity of $81.06 \%)$, which was greater than MKL (74.77\%), JRC (73.83\%), mSRC (75.70\%), and mSCDDL (77.66\%). The comparison of the ROC curves for classification of $\mathrm{MCI}$ and $\mathrm{CU}$ are shown in Figure 3B and the comparison of AUCs is shown in Table 3. The ROC curve of MKSCDDL was closer to the top-left corner than that of MKL, JRC, mSRC, and mSCDDL. Further, based on significant validation, MKSCDDL was numerically better than the corresponding methods with AUC, which was 0.839 for the MKSCDDL method compared with that of the corresponding methods (AUC $=0.804, p=0.534$ for $\mathrm{MKL}$; $\mathrm{AUC}=0.793$, $p=0.331$ for JRC; $\mathrm{AUC}=0.785, p=0.223$ for $\mathrm{mSRC}$; and

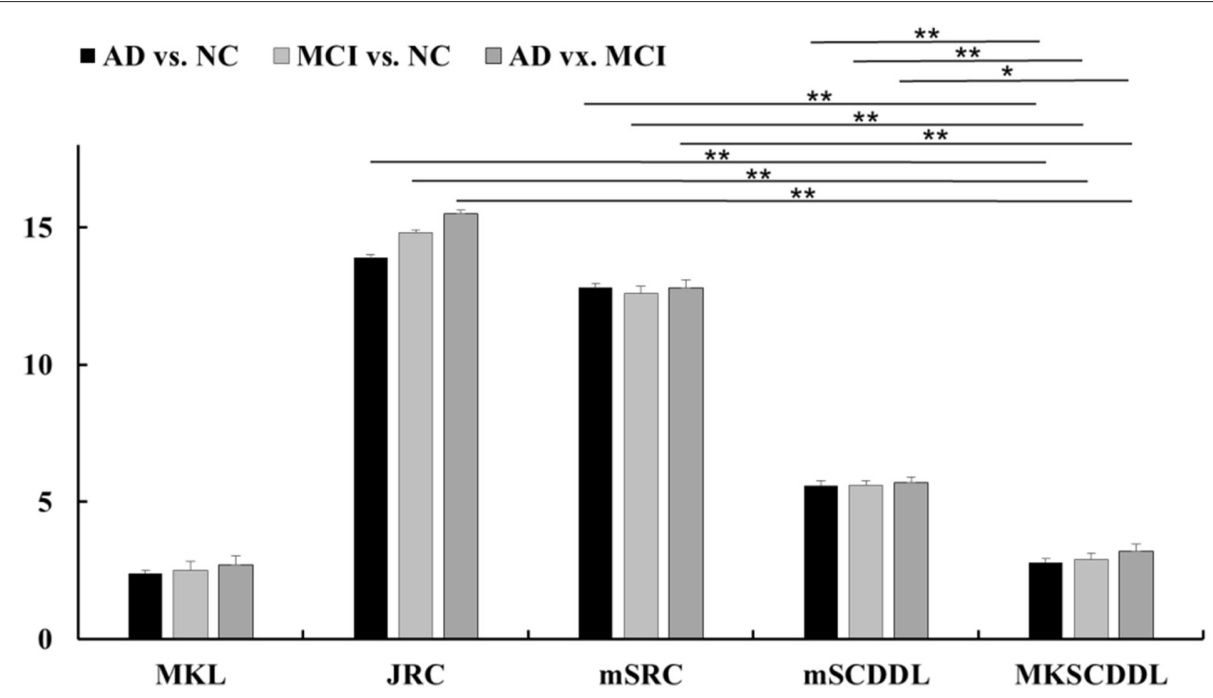

FIGURE 5 | Comparison of testing time of different multi-modality methods for classification AD, MCl, and CU based on MKL, JRC, mSRC, mSCDDL, and MKSCDDL (**indicates $0.01 \leq p<0.05$; *indicates $0.05 \leq p<0.10$ ). 
$\mathrm{AUC}=0.828, p=0.843$ for $\mathrm{mSCDDL}$ ), as shown in Figure 4B. As shown in Figure 5, MKSCDDL consumed much less testing time than JRC $(p=0.009), \operatorname{mSRC}(p=0.015)$ and mSCDDL $(p=0.047)$, and was comparable with the MKL $(p=0.389)$ method.

For classifying AD from MCI, MKSCDDL achieved an accuracy of $74.47 \%$ (with sensitivity of $72.44 \%$ and specificity of $78.99 \%)$, which was greater than MKL (72.94\%), JRC (72.05\%), mSRC (68.55\%), and mSCDDL (73.20\%). The comparison of the ROC curves for classification of $\mathrm{AD}$ and $\mathrm{MCI}$ are shown in Figure 3C and the comparison of AUCs is shown in Table 3. The ROC curve of MKSCDDL was closer to the top-left corner than that of MKL, JRC, mSRC, and mSCDDL. Further, based on significant validation, MKSCDDL was numerically better than the corresponding methods with AUC, which was 0.791 for the MKSCDDL method compared with that of the corresponding methods $(\mathrm{AUC}=0.779, p=0.600$ for $\mathrm{MKL}$; $\mathrm{AUC}=0.772$, $p=0.477$ for JRC; $\mathrm{AUC}=0.693, p=0.120$ for $\mathrm{mSRC}$; and $\mathrm{AUC}=0.780, p=0.593$ for $\mathrm{mSCDDL}$ ), which shown in Figure 4C. As shown in Figure 5, MKSCDDL consumed much less testing time than JRC $(p=0.011), \operatorname{mSRC}(p=0.019)$ and mSCDDL $(p=0.059)$, and was comparable with the MKL $(p=0.352)$ method.

\section{Biomarkers for $\mathrm{AD}, \mathrm{MCl}$, and $\mathrm{CU}$ Classification}

To characterize the classification performance for $\mathrm{AD}, \mathrm{MCI}$, and CU with all 90 features (without feature selection), the classification accuracy has been investigated under feature selection with $1,2,3, \ldots$, or 90 features for each of the ranked 90 features. The results of classification performance for different numbers of ranked features are shown in Figure 6.

The figure shows that the MKSCDDL method could reach strong classification accuracy even with fewer than 5 features (the top 5\% ranked features on sMRI, FDG-PET, and florbetapir$\mathrm{PET}$ ) for $\mathrm{AD} / \mathrm{MCI} / \mathrm{CU}$ classification. In particular, there was higher than $90 \%$ accuracy for classifying $\mathrm{AD}$ from $\mathrm{CU}$, higher than $78 \%$ accuracy for distinguishing $\mathrm{MCI}$ from $\mathrm{CU}$, and higher than 61\% accuracy for discriminating $\mathrm{AD}$ and MCI. The MKSCDDL method was stable (with less ups and downs) for the classification of $\mathrm{AD} / \mathrm{MCI}$ from $\mathrm{CU}$, which indicated that redundant features likely introduced little interference of classification. For classification of $\mathrm{AD}$ and MCI, though the accuracy was also acceptable, it was not as stable as the classification accuracy for $\mathrm{AD} / \mathrm{MCI}$ with $\mathrm{CU}$, which may be due to the biomarkers for $\mathrm{AD}$ and MCI having very high similarity. When the top $10 \%$ features were used, the accuracy for classification of $\mathrm{AD}$ and MCI was higher than $64 \%$.

As shown in Figure 6, the MKSCDDL could achieve a promising or acceptable accuracy even with less than 5 features (the top 5\% ranked features). Thus, for convenience, one could apply a small set of features to effectively discriminate AD, MCI, and CU. Here, the top 5-10\% ranked features (4-9 features) consisted of sMRI, FDG-PET, and florbetapir-PET data and could be chosen as biomarkers for further classification (Xu et al., 2016). 


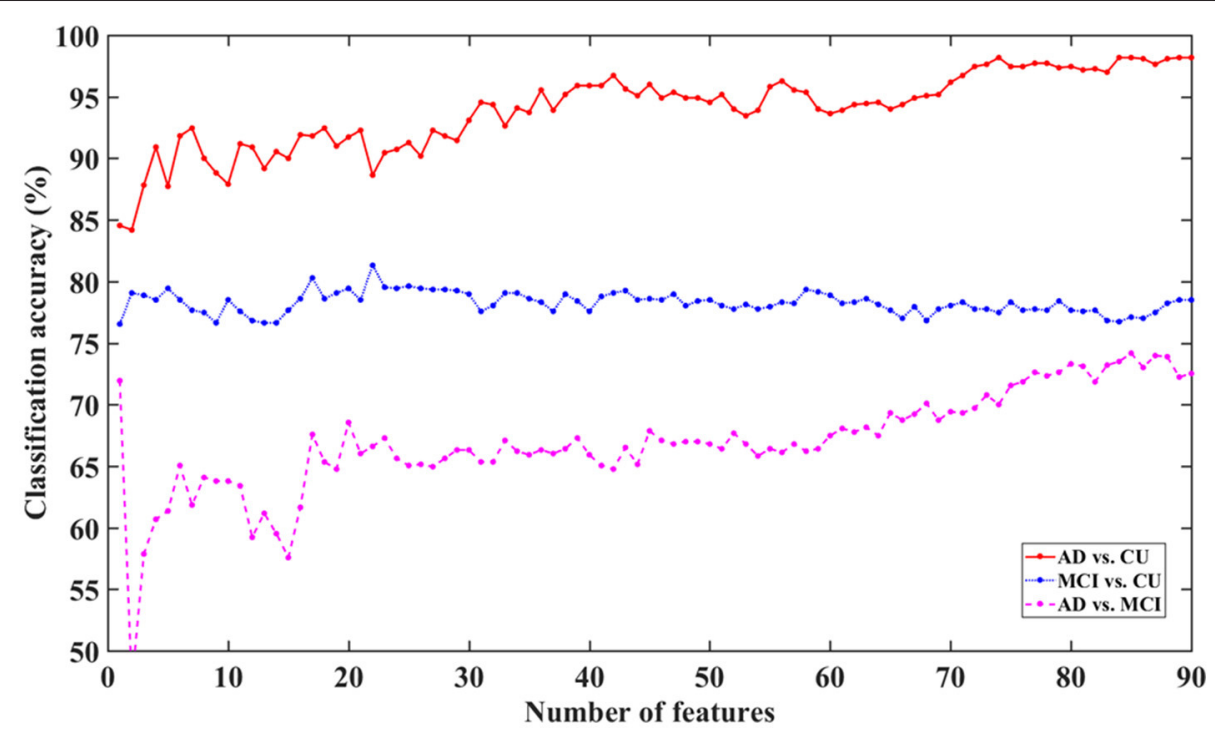

FIGURE 6 | Classification accuracy for AD, MCl, and $\mathrm{CU}$ with different feature dimensions based on MKSCDDL.

The biomarkers of different modalities for classification of the AD, MCI, and CU groups are displayed in Table 4 and Figure 7. For classification of $\mathrm{AD}$ and $\mathrm{CU}$, the Hippocampus, Inferior Temporal, and ParaHippocampal may be the discriminating biomarkers on sMRI; the Angular, Posterior Cingulum, and Inferior Parietal may be the important regions on FDG-PET; and the Hippocampus and ParaHippocampal may be the key regions on florbetapir-PET. For discriminating MCI from CU, the Hippocampus, Middle Temporal, and ParaHippocampal may be the discriminating biomarkers on sMRI; the Angular and Posterior Cingulum may be the important regions on FDGPET; and the Hippocampus, Posterior Cingulum, and Middle Frontal (Orbital part) may be the key regions on florbetapirPET. For differentiating AD and MCI, the SupraMarginal, Angular, and left Superior Frontal (Orbital part) were the discriminating biomarkers on sMRI; the Angular, Inferior Parietal, and SupraMarginal may be the important regions on FDG-PET; and the Calcarine, Heschl, and Lingual may be the key regions on florbetapir-PET.

For AD and CU classification, the Hippocampus (Wisse et al., 2014; de Flores et al., 2015; Voineskos et al., 2015), Inferior Temporal (Seo et al., 2017), ParaHippocampal (Guo et al., 2014; Peng et al., 2016), Angular (Sanabria-Diaz et al., 2013), Posterior Cingulum (Nakata et al., 2009; Demirhan et al., 2015), and Inferior Parietal (Murray et al., 2015; Zhang et al., 2015) have been proposed in several studies to be effective biomarkers. The Hippocampus (Wee et al., 2011; Zhou et al., 2011; Liu et al., 2014a), Middle Temporal (Lenzi et al., 2011; Jiang et al., 2014), ParaHippocampal (Cerami et al., 2015; Kato et al., 2016), Angular (Nobili et al., 2010; Martlno et al., 2013; Zu et al., 2015), Posterior Cingulum (Choo et al., 2010; Yu et al., 2017), and Middle Frontal (Orbital part) (Xiang et al., 2013) have been reported as the important regions for discriminating MCI and CU. For differentiating AD and MCI, the SupraMarginal (Esposito et al.,
TABLE 4 | The most discriminating regions for classification $A D, M C l$, and $C U$ based on SMRI, FDG-PET, and florbetapir-PET.

\begin{tabular}{|c|c|c|}
\hline sMRI & FDG-PET & Florbetapir PET \\
\hline \multicolumn{3}{|l|}{ AD vs. CU } \\
\hline Left hippocampus & Left angular & Left hippocampus \\
\hline Right hippocampus & $\begin{array}{l}\text { Left posterior } \\
\text { cingulum }\end{array}$ & Right hippocampus \\
\hline Left inferior temporal & Right angular & Left parahippocampal \\
\hline Right parahippocampal & Right inferior parietal & Right parahippocampal \\
\hline \multicolumn{3}{|l|}{$\mathrm{MCl}$ vs. CU } \\
\hline Left hippocampus & $\begin{array}{l}\text { Left posterior } \\
\text { cingulum }\end{array}$ & Left hippocampus \\
\hline Right hippocampus & Left angular & Right hippocampus \\
\hline Left middle temporal & $\begin{array}{l}\text { Right posterior } \\
\text { cingulum }\end{array}$ & Right posterior cingulum \\
\hline Right parahippocampal & Right angular & Left middle frontal (orbital part) \\
\hline \multicolumn{3}{|l|}{ AD vs. $\mathrm{MCl}$} \\
\hline Left supramarginal & Right angular & Left calcarine \\
\hline Right angular & Left angular & Left heschl \\
\hline Right supramarginal & Right inferior parietal & Right lingual \\
\hline $\begin{array}{l}\text { Left superior frontal } \\
\text { (orbital part) }\end{array}$ & Left supramarginal & Right heschl \\
\hline
\end{tabular}

2013; Moretti, 2015), Angular (Hirao et al., 2005; Griffith et al., 2010; Li et al., 2016), Superior Frontal (Orbital part) (Liu et al., 2012), Inferior Parietal (Desikan et al., 2009; Triplett et al., 2016), Calcarine (Liu et al., 2012), Heschl (Hanggi et al., 2011), and Lingual (Li et al., 2016) may be the key biomarkers for diagnosis.

Therefore, MKSCDDL was proved as a very efficient method for classifying $\mathrm{AD}$ or $\mathrm{MCI}$ from $\mathrm{CU}$, and had potential to discriminate $\mathrm{AD}$ from $\mathrm{MCI}$, as compared to the single-modality 




FIGURE 7 | Biomarkers with sMRI, FDG-PET, and florbetapir-PET (A) for classification AD and CU; (B) for classification MCl and CU; and (C) for classification AD and $\mathrm{MCl}$.

method and several state-of-art multi-modality methods. The MKSCDDL method performed better than MKL, JRC, mSRC, and mSCDDL in terms of accuracy rate and AUC, often significantly on validation but at least numerically for AD, MCI, and CU classification. In addition, the MKSCDDL method took less computation time than did JRC, mSRC, and MSCDDL, and was comparable to MKL in terms of computation time. Together, this indicates that the MKSCDDL method could potentially play an important role in $\mathrm{AD}$ and $\mathrm{MCI}$ diagnosis.

\section{CONCLUSIONS}

In this study, a novel DL method, named as MKSCDDL with previous successful application to face recognition, was introduced combining sMRI, FDG-PET, and florbetapir-PET for differentiating $\mathrm{AD}, \mathrm{MCI}$, and $\mathrm{CU}$. The results suggested that the MKSCDDL is promising for classification and diagnose diseases with neuroimaging data. 


\section{ETHICS STATEMENT}

All procedures performed in studies involving human participants were in accordance with the ethical standards of the institutional and/or national research committee and with the 1964 Helsinki declaration and its later amendments or comparable ethical standards.

\section{Informed Consent}

Informed consent was obtained from all individual participants included in the study.

\section{AUTHOR CONTRIBUTIONS}

XW, LY: designed the study. LX, KC: collected the original imaging data. QL, XW, KC: managed and analyzed the imaging data. QL and XW: wrote the manuscript. All authors contributed to and have approved the final manuscript.

\section{FUNDING}

This work was supported by the Funds for International Cooperation and Exchange of the National Natural Science

\section{REFERENCES}

Ashburner, J. (2007). A fast diffeomorphic image registration algorithm. Neuroimage 38, 95-113. doi: 10.1016/j.neuroimage.2007.07.007

Asif, M. S., and Romberg, J. (2010). Dynamic updating for L1 minimization. IEEE J. Select. Top. Signal Process. 4, 421-434. doi: 10.1109/JSTSP.2009.2039174

Camus, V., Payoux, P., Barré, L., Desgranges, B., Voisin, T., Tauber, C., et al. (2012). Using PET with 18F-AV-45 (florbetapir) to quantify brain amyloid load in a clinical environment. Eur. J. Nucl. Med. Mol. Imaging 39, 621-631. doi: 10.1007/s00259-011-2021-8

Candes, E., and Romberg, J. (2005). l1-MAGIC: Recovery of Sparse Signals via Convex Programming. Available online at: www.acm.caltech.edu/l1magic/ downloads/l1magic.pdf

Cerami, C., Della Rosa, P. A., Magnani, G., Santangelo, R., Marcone, A., Cappa, S. F., et al. (2015). Brain metabolic maps in Mild Cognitive Impairment predict heterogeneity of progression to dementia. Neuroimage Clin. 7(Suppl. C), 187-194. doi: 10.1016/j.nicl.2014.12.004

Choo, I. H., Lee, D. Y., Oh, J. S., Lee, J. S., Lee, D. S., Song, I. C., et al. (2010). Posterior cingulate cortex atrophy and regional cingulum disruption in mild cognitive impairment and Alzheimer's disease. Neurobiol. Aging 31, 772-779. doi: 10.1016/j.neurobiolaging.2008.06.015

de Flores, R., La Joie, R., and Chételat, G. (2015). Structural imaging of hippocampal subfields in healthy aging and Alzheimer's disease. Neuroscience 309, 29-50. doi: 10.1016/j.neuroscience.2015.08.033

Demirhan, A., Nir, T. M., Zavaliangos-Petropulu, A., Jack, C. R., Weiner, M. W., Bernstein, M. A., et al. (2015). Feature selection improves the accuracy of classifying alzheimer disease using diffusion tensor images. Proc. IEEE Int. Symp. Biomed. Imaging 2015, 126-130. doi: 10.1109/ISBI.2015. 7163832

Desikan, R. S., Cabral, H. J., Fischl, B., Guttmann, C. R. G., Blacker, D., and Hyman, B. T. (2009). Temporoparietal MR imaging measures of atrophy in subjects with mild cognitive impairment that predict subsequent diagnosis of Alzheimer Disease. Am. J. Neuroradiol. 30, 532-538. doi: 10.3174/ajnr.A1397

Dyrba, M., Grothe, M., Kirste, T., and Teipel, S. J. (2015). Multimodal analysis of functional and structural disconnection in Alzheimer's disease using multiple kernel SVM. Hum. Brain Mapp. 36, 2118-2131. doi: 10.1002/hbm. 22759
Foundation of China [grant number 61210001], the General Program of National Natural Science Foundation of China [grant number 61571047], the Fundamental Research Funds for the Central Universities [grant number 2017STUD34], and the Fundamental Research Funds for the Central Universities [grant number 2017EYT36].

\section{ACKNOWLEDGMENTS}

The data set used in preparation of this paper was obtained from the Alzheimer's Disease Neuroimaging Initiative (ADNI) database (adni.loni.ucla.edu). As such, the investigators within the ADNI contributed to the design and implementation of $\mathrm{ADNI}$ and/or provided data but did not participate in the analysis or writing of this report. A complete listing of ADNI investigators can be found at: https://adni.loni.usc.edu/wp-content/uploads/ how_to_apply/ADNI_Data_Use_Agreement.pdf.

\section{SUPPLEMENTARY MATERIAL}

The Supplementary Material for this article can be found online at: https://www.frontiersin.org/articles/10.3389/fncom. 2017.00117/full\#supplementary-material

Esposito, R., Mosca, A., Pieramico, V., Cieri, F., Cera, N., and Sensi, S. L. (2013) Characterization of resting state activity in MCI individuals. PeerJ. 1:e135. doi: $10.7717 /$ peerj.135

Figueiredo, M. A., Nowak, R. D., and Wright, S. J. (2007). Gradient projection for sparse reconstruction: application to compressed sensing and other inverse problems. IEEE J. Sel. Top. Signal Process. 1, 586-597. doi: 10.1109/JSTSP.2007.910281

Gao, Y., Tan, M. S., Wang, H. F., Zhang, W., Wang, Z. X., Jiang, T., et al. (2016). ZCWPW is associated with late-onset Alzheimer's disease in Han Chinese: a replication study and meta-analyses. Oncotarget 7, 20305-20311. doi: 10.18632/oncotarget.7945

Garcés, P., Pineda-Pardo, J. A., Canuet, L., Aurtenetxe, S., Lopez, M. E., Marcos, A., et al. (2014). The default mode network is functionally and structurally disrupted in amnestic mild cognitive impairment - A bimodal MEG-DTI study. Neruoimage Clin. 6, 214-221. doi: 10.1016/j.nicl.2014.09.004

Gönen, M., and Alpaydin, E. (2011). Multiple kernel learning algorithms. J. Mach. Learn. Res. 12, 2211-2268. Available online at: http://www.jmlr.org/papers/ volume12/gonen11a/gonen11a.pdf

Griffith, H. R., Stewart, C. C., Stoeckel, L. E., Okonkwo, O. C., den Hollander, J. A., Martin, R. C., et al. (2010). MRI volume of the angular gyri predicts financial skill deficits in patients with amnestic mild cognitive impairment. J. Am. Geriatr. Soc. 58, 265-274. doi: 10.1111/j.1532-5415.2009.02679.x

Guo, Y., Zhang, Z., Zhou, B., Wang, P., Yao, H., Yuan, M., et al. (2014). Grey-matter volume as a potential feature for the classification of Alzheimer's disease and mild cognitive impairment: an exploratory study. Neurosci. Bull. 30, 477-489. doi: 10.1007/s12264-013-1432-x

Hanggi, J., Streffer, J., Jancke, L., and Hock, C. (2011). Volumes of lateral temporal and parietal structures distinguish between healthy aging, mild cognitive impairment, and Alzheimer's disease. J. Alzheimers Dis. 26, 719-734. doi: 10.3233/JAD-2011-101260

Harandi, M., and Salzmann, M. (2015). "Riemannian coding and dictionary learning: kernels to the rescue," in 2015 IEEE Conference on Computer Vision and Pattern Recognition (CVPR) (Boston, MA).

Hirao, K., Ohnishi, T., Hirata, Y., Yamashita, F., Mori, T., Moriguchi, Y., et al. (2005). The prediction of rapid conversion to Alzheimer's disease in mild cognitive impairment using regional cerebral blood flow SPECT. Neuroimage 28, 1014-1021. doi: 10.1016/j.neuroimage.2005.06.066 
Hussain, M., Wajid, S. K., Elzaart, A., and Berbar, M. (2011). “A comparison of SVM kernel functions for breast cancer detection, in Computer Graphics," in 2011 Eighth International Conference on Imaging and Visualization (CGIV) (Singapore).

Jiang, X., Zhu, D., Li, K., Zhang, T., Wang, L., Shen, D., et al. (2014). Predictive models of resting state networks for assessment of altered functional connectivity in mild cognitive impairment. Brain Imaging Behav. 8, 542-557. doi: $10.1007 / \mathrm{s} 11682-013-9280-\mathrm{x}$

Jiang, Z., Lin, Z., and Davis, L. S. (2013). Label consistent K-SVD: learning a discriminative dictionary for recognition. IEEE Trans. Pattern Anal. Mach. Intell. 35, 2651-2664. doi: 10.1109/TPAMI.2013.88

Kato, T., Inui, Y., Nakamura, A., and Ito, K. (2016). Brain fluorodeoxyglucose (FDG) PET in dementia. Ageing Res. Rev. 30(Suppl. C), 73-84. doi: 10.1016/j.arr.2016.02.003

Langbaum, J. B. S., Chen, K., Lee, W., Reschke, C., Bandy, D., Fleisher, A. S., et al. (2009). Categorical and correlational analyses of baseline fluorodeoxyglucose positron emission tomography images from the Alzheimer's Disease Neuroimaging Initiative (ADNI). Neuroimage 45, 1107-1116. doi: 10.1016/j.neuroimage.2008.12.072

Lenzi, D., Serra, L., Perri, R., Pantano, P., Lenzi, G. L., Paulesu, E., et al. (2011). Single domain amnestic MCI: A multiple cognitive domains fMRI investigation. Neurobio. Aging 32, 1542-1557. doi: 10.1016/j.neurobiolaging. 2009.09.006

Li, Q., Wu, X., Xu, L., Chen, K., Yao, L., and Li, R. (2017). Multimodal discriminative dictionary learning for Alzheimer's disease and mild cognitive impairment. Comput. Methods Programs Biomed. 150, 1-8. doi: 10.1016/j.cmpb.2017.07.003

Li, Y., Wang, X., Li, Y., Sun, Y., Sheng, C., Li, H., et al. (2016). Abnormal resting-state functional connectivity strength in mild cognitive impairment and its conversion to Alzheimer's Disease. Neural Plast. 2016:4680972. doi: $10.1155 / 2016 / 4680972$

Liu, F., Suk, H. I., Wee, C. Y., Chen, H., and Shen, D. (2013). Highorder graph matching based feature selection for Alzheimer's Disease identification. Med. Image Comput. Comput Assist. Interv. 16(Pt 2), 311-318. doi: 10.1007/978-3-642-40763-5_39

Liu, F., Wee, C. Y., Chen, H., and Shen, D. (2014b). Inter-modality relationship constrained multi-modality multi-task feature selection for Alzheimer's Disease and mild cognitive impairment identification. Neuroimage 84, 466-475. doi: 10.1016/j.neuroimage.2013.09.015

Liu, F., Zhou, L., Shen, C., and Yin, J. (2014a). Multiple kernel learning in theprimal for multimodal Alzheimer's disease classification. IEEE J. Biomed. Health Inform. 18, 984-990. doi: 10.1109/JBHI.2013.2285378

Liu, Z., Zhang, Y., Yan, H., Bai, L., Dai, R., Wei, W., et al. (2012). Altered topological patterns of brain networks in mild cognitive impairment and Alzheimer's disease: a resting-state fMRI study. Psychiatry Res. Neuroimaging 202, 118-125. doi: 10.1016/j.pscychresns.2012.03.002

Manevitz, L. M., and Yousef, M. (2001). One-class SVMs for document classification. J. Mach. Learn. Res. 2, 139-154. Available online at: http://www. jmlr.org/papers/volume2/manevitz01a/manevitz01a.pdf

Martlno, M. E., de Villoria, J. G., Lacalle-Aurioles, M., Olazarán, J., Cruz, I., Navarro, E., et al. (2013). Comparison of different methods of spatial normalization of FDG-PET brain images in the voxel-wise analysis of MCI patients and controls. Ann. Nucl. Med. 27, 600-609. doi: 10.1007/s12149-013-0723-7

Moretti, D. V. (2015). Theta and alpha EEG frequency interplay in subjects with mild cognitive impairment: evidence from EEG, MRI, and SPECT brain modifications. Front. Aging Neurosci. 7:31. doi: 10.3389/fnagi.2015.00031

Murray, M. E., Lowe, V. J., Graff-Radford, N. R., Liesinger, A. M., Cannon, A., Przybelski, S. A., et al. (2015). Clinicopathologic and 11C-Pittsburgh compound B implications of Thal amyloid phase across the Alzheimer's disease spectrum. Brain 138, 1370-1381. doi: 10.1093/brain/awv050

Nakata, Y., Sato, N., Nemoto, K., Abe, O., Shikakura, S., Arima, K., et al. (2009). Diffusion abnormality in the posterior cingulum and hippocampal volume: correlation with disease progression in Alzheimer's disease. Magn. Reson. Imaging 27, 347-354. doi: 10.1016/j.mri.2008.07.013

Nguyen, H., Patel, V. M., Nasrabadi, N. M., and Chellappa, R. (2012). "Kernel dictionary learning, in Acoustics," in 2012 IEEE International Conference on Speech and Signal Processing (ICASSP) (Kyoto), 2021-2024.
Nobili, F., Mazzei, D., Dessi, B., Morbelli, S., Brugnolo, A., Barbieri, P., et al. (2010). Unawareness of memory deficit in amnestic MCI: FDGPET findings. J. Alzheimers Dis. 22, 993-1003. doi: 10.3233/JAD-2010100423

Peng, J., An, L., Zhu, X., Jin, Y., and Shen, D. (2016). Structured sparse kernel learning for imaging genetics based Alzheimer's Disease diagnosis. Med. Image Comput. Comput. Assist. Interv. 9901, 70-78. doi: 10.1007/978-3-319-46723-8_9

Petersen, R. C. (2004). Mild cognitive impairment as a diagnostic entity. J. Intern. Med. 256, 183-194. doi: 10.1111/j.1365-2796.2004.01388.x

Petersen, R. C., Stevens, J. C., Ganguli, M., Tangalos, E. G., Cummings, J. L., and DeKosky, S. T. (2001). Practice parameter: early detection of dementia: mild cognitive impairment (an evidence-based review) Report of the Quality Standards Subcommittee of the American Academy of Neurology. Neurology 56, 1133-1142. doi: 10.1212/WNL.56.9.1133

Pham, N., and Pagh, R. (2013). "Fast and scalable polynomial kernels via explicit feature maps," in Proceedings of the 19th ACM SIGKDD International Conference on Knowledge Discovery and Data Mining (Chicago, IL), 239-247.

Rajapakse, J. C., Giedd, J. N., and Rapoport, J. L. (1997). Statistical approach to segmentation of single-channel cerebral MR images. IEEE Trans. Med. Imaging 16, 176-186. doi: 10.1109/42.563663

Reitan, R. (1958). Validity of the Trail Making Test as an indicator of organic brain damage. Percept. Mot. Skills 8, 271-276. doi: 10.2466/pms.1958.8.3.271

Sabbagh, M. N., Chen, K., Rogers, J., Fleisher, A. S., Liebsack, C., and Bandy, D. (2015). Florbetapir PET, FDG PET, and MRI in Down syndrome individuals with and without Alzheimer's dementia. Alzheimers Dement. 11, 994-1004. doi: 10.1016/j.jalz.2015.01.006

Saint-Aubert, L., Barbeau, E. J., Péran, P., Nemmi, F., Vervueren, C., Mirabel, H., et al. (2013). Cortical florbetapir-PET amyloid load in prodromal Alzheimer's disease patients. EJNMMI Res. 3:43. doi: 10.1186/2191-219X-3-43

Sanabria-Diaz, G., Martinez-Montes, E., and Melie-Garcia, L. (2013). Glucose metabolism during resting state reveals abnormal brain networks organization in the Alzheimer's Disease and mild cognitive impairment. PLoS ONE 8:e68860. doi: 10.1371/journal.pone.0068860

Schölkopf, B., Herbrich, R., and Smola, A. J. (2001). “A generalized representer theorem," in International Conference on Computational Learning Theory (Amsterdam), 416-426.

Selkoe, D. J., and Hardy, J. (2016). The amyloid hypothesis of Alzheimer's disease at 25 years. EMBO Mol. Med. 8, 595-608. doi: 10.15252/emmm.201606210

Seo, S. W., Ayakta, N., Grinberg, L. T., Villeneuve, S., Lehmann, M., Reed, B., et al. Rabinovici, G. D. (2017). Regional correlations between [11C]PIB PET and post-mortem burden of amyloid-beta pathology in a diverse neuropathological cohort. Neuroimage Clin. 13(Suppl. C), 130-137. doi: 10.1016/j.nicl.2016.11.008

Sonnenburg, S., Rätsch, G., Schäfer, C., and Schölkopf, B. (2006). Large scale multiple kernel learning. J. Mach. Learn. Res. 7, 1531-1565. Available online at: http://www.jmlr.org/papers/volume7/sonnenburg06a/sonnenburg06a.pdf

Suk, H. I., Lee, S. W., and Shen, D. G. (2015). Latent feature representation with stacked auto-encoder for AD/MCI diagnosis. Brain Struct. Funct. 220, 841-859. doi: 10.1007/s00429-013-0687-3

Suk, H. I., Wee, C. Y., and Shen, D. (2013). "Discriminative group sparse representation for mild cognitive impairment classification," in International Workshop on Machine Learning in Medical Imaging (Nagoya), 8184, 131-138.

Tan, M. S., Yu, J. T., Jiang, T., Zhu, X. C., Wang, H. F., Zhang, W., et al. (2013). NLRP3 polymorphisms are associated with late-onset Alzheimer's disease in Han Chinese. J. Neuroimmunol. 265, 91-95. doi: 10.1016/j.jneuroim.2013.10.002

Tohka, J., Zijdenbos, A., and Evans, A. (2004). Fast and robust parameter estimation for statistical partial volume models in brain MRI. Neuroimage 23 , 84-97. doi: 10.1016/j.neuroimage.2004.05.007

Triplett, J. C., Swomley, A., M., Cai, J., and Klein, J. B., Butterfield, D. A. (2016) Quantitative phosphoproteomic analyses of the inferior parietal lobule from three different pathological stages of Alzheimer's Disease. J. Alzheimers Dis. 49, 45-62. doi: 10.3233/JAD-150417

Tzourio-Mazoyer, N., Landeau, B., Papathanassiou, D., et al. (2002). Automated anatomical labeling of activations in SPM using a macroscopic anatomical parcellation of the MNI MRI single-subject brain. Neuroimage 15, 273-289. doi: $10.1006 /$ nimg.2001.0978 
Voineskos, A. N., Winterburn, J. L., Felsky, D., Pipitone, J., Rajji, T. K., and Mulsant, B., H., et al. (2015). Hippocampal (subfield) volume and shape in relation to cognitive performance across the adult lifespan. Hum. Brain Mapp. 36, 3020-3037. doi: 10.1002/hbm.22825

Wang, P., Chen, K., Yao, L., Hu, B., Wu, X., Zhang, J., et al. (2016). Alzheimer's Disease neuroimaging initiative, multimodal classification of mild cognitive impairment based on partial least squares. J. Alzheimers Dis. 54, 359-371. doi: $10.3233 / J A D-160102$

Wee, C. Y., Yap, P. T., Li, W., Denny, K., Browndyke, J. N., Potter, G. G., et al. (2011). Enriched white-matter connectivity networks for accurate identification of mci patients. Neuroimage 54, 1812-1822. doi: 10.1016/j.neuroimage.2010.10.026

Wee, C. Y., Yap, P. T., Zhang, D., Denny, K., Browndyke, J. N., Potter, G. G., et al. (2012). Identification of MCI individuals using structural and functional connectivity networks. Neuroimage 59, 2045-2056. doi: 10.1016/j.neuroimage.2011.10.015

Wei, R., Li, C., Fogelson, N., Li, L., and Alzheimer's Disease Neuroimaging Initiative (2016). Prediction of conversion from mild cognitive impairment to Alzheimer's Disease using MRI and structural network features. Front. Aging Neurosci. 8:76. doi: 10.3389/fnagi.2016.00076

Westman, E., Muehlboeck, J., and Simmons, A. (2012). Combining MRI and CSF measures for classification of Alzheimer's disease and prediction of mild cognitive impairment conversion. Neuroimage 62, 229-238. doi: 10.1016/j.neuroimage.2012.04.056

Wisse, L. E., Biessels, G. J., Heringa, S. M., Kuijjf, H. J., Koek, D. K., Luijten, P. R., et al. (2014). Hippocampal subfield volumes at 7T in early Alzheimer's disease and normal aging. Neurobiol. Aging 35, 2039-2045. doi: 10.1016/j.neurobiolaging.2014.02.021

Wu, X., Li, Q., Xu, L., Chen, K., and Yao, L. (2017). Multi-feature kernel discriminant dictionary learning for face recognition. Pattern Recogn. 66, 404-411. doi: 10.1016/j.patcog.2016.12.001

Xiang, J., Guo, H., Cao, R., Liang, H., and Chen, J. (2013). An abnormal restingstate functional brain network indicates progression towards Alzheimer's disease. Neural Regen. Res. 8, 2789-2799. doi: 10.3969/j.issn.1673-5374. 2013.30.001

Xu, L., Wu, X., Chen, K., and Yao, L. (2015). Multi-modality sparse representation-based classification for Alzheimer's disease and mild cognitive impairment. Comput. Methods Programs Biomed. 122, 182-190. doi: 10.1016/j.cmpb.2015.08.004

Xu, L., Wu, X., Li, R., Chen, K., Long, Z., Zhang, J., et al. (2016). Prediction of progressive mild cognitive impairment by multi-modal neuroimaging biomarkers. J. Alzheimers Dis. 51, 1045-1056. doi: 10.3233/JAD151010

Yang, M., Zhang, L., Feng, X., and Zhang, D. (2014). Sparse representation based fisher discrimination dictionary learning for image classification. Int. J. Comput. Vis. 19, 209-232. doi: 10.1007/s11263-0140722-8

Yu, E., Liao, Z., Mao, D., Zhang, Q., Ji, G., Li, Y., et al. (2017). Directed functional connectivity of posterior cingulate cortex and whole brain in Alzheimer's Disease and mild cognitive impairment. Curr. Alzheimer Res. 14, 628-635. doi: 10.2174/1567205013666161201201000
Zhang, D., Shen, D., and Alzheimer's Disease Neuroimaging Initiative (2012a) Multi-modal multi-task learning for joint prediction of multiple regression and classification variables in Alzheimer's disease. Neuroimage 59, 895-907. doi: 10.1016/j.neuroimage.2011.09.069

Zhang, D., Shen, D., and Alzheimer's Disease Neuroimaging Initiative (2012b). Predicting future clinical changes of MCI patients using longitudinal and multimodal biomarkers. PLOS ONE 7:e33182. doi: 10.1371/journal. pone.0033182

Zhang, D., Wang, Y., Zhou, L., Yuan, H., Shen, D., and Alzheimer's Disease Neuroimaging Initiative (2011). Multimodal classification of Alzheimer's disease and mild cognitive impairment. Neuroimage 55, 856-867. doi: 10.1016/j.neuroimage.2011.01.008

Zhang, Q., and Li, B. (2010). "Discriminative K-SVD for dictionary learning in face recognition," in IEEE Conference on Computer Vision and Pattern Recognition (CVPR) (San Francisco, CA), 2691-2698.

Zhang, S., Smailagic, N., Hyde, C., Noel-Storr, A. H., Takwoingi, Y., McShane, R., et al. (2014). C-PIB-PET for the early diagnosis of Alzheimer's disease dementia and other dementias in people with mild coginitive impairment(MCI). Cochrane Database Syst. Rev. 7:CD010386. doi: 10.1002/14651858.CD010386.pub2

Zhang, Y., Dong, Z., Phillips, P., Wang, S., Ji, G., Yang, J., et al. (2015). Detection of subjects and brain regions related to Alzheimer's disease using 3D MRI scans based on eigenbrain and machine learning. Front. Comput. Neurosci. 9:66. doi: 10.3389/fncom.2015.00066

Zhou, L., Wang, Y., Li, Y., Yap, P. T., and Shen, D. (2011). Hierarchical anatomical brain networks for MCI prediction: revisiting volumetric measures. PLoS ONE 6:e21935. doi: 10.1371/journal.pone.0021935

Zhu, X., Suk, H., and Shen, D. (2014a). A novel matrix-similarity based loss function for joint regression and classification in $\mathrm{AD}$ diagnosis. Neuroimage 100, 91-105. doi: 10.1016/j.neuroimage.2014.05.078

Zhu, X., Suk, H., and Shen, D. (2014b). A novel multi-relation regularization method for regression and classification in AD diagnosis. Med. Image Comput. Comput. Assist. Interv. 17, 401-408. doi: 10.1007/978-3-319-10443-0_51

Zou, H., and Hastie, T. (2005). Regularization and variable selection via the elastic net. J. R. Stat. Soc. 67, 301-320. doi: 10.1111/j.1467-9868.2005.00503.x

Zu, C., Jie, B., Liu, M., Chen, S., Shen, D., Zhang, D., et al. (2015). Labelaligned multi-task feature learning for multimodal classification of Alzheimer's disease and mild cognitive impairment. Brain Imaging Behav. 10, 1148-1159. doi: 10.1007/s11682-015-9480-7

Conflict of Interest Statement: The authors declare that the research was conducted in the absence of any commercial or financial relationships that could be construed as a potential conflict of interest.

Copyright (c) $2018 \mathrm{Li}, \mathrm{Wu}, \mathrm{Xu}$, Chen, Yao and Alzheimer's Disease Neuroimaging Initiative. This is an open-access article distributed under the terms of the Creative Commons Attribution License (CC BY). The use, distribution or reproduction in other forums is permitted, provided the original author(s) or licensor are credited and that the original publication in this journal is cited, in accordance with accepted academic practice. No use, distribution or reproduction is permitted which does not comply with these terms. 